\title{
A Realistic Path Loss Model for Real-time Communication in the Urban Grid Environment for Vehicular Ad hoc Networks
}

\author{
Ehsan Mostajeran ${ }^{1}$, Rafidah Md Noor ${ }^{1^{*}}$, Mohammad Hossein Anisi ${ }^{2 *}$, Ismail Ahmedy ${ }^{1}$ and \\ Fawad Ali Khan ${ }^{1}$ \\ ${ }^{\mathbf{1}}$ Department of Computer System and Technology, Faculty of Computer Science and Information Technology, \\ University of Malaya, Kuala Lumpur, 50603, Malaysia. \\ [e-mail: ehsan_mostajeran@siswa.um.edu.my, fidah@um.edu.my, ismailahmedy@um.edu.my, \\ fawadkn@siswa.um.edu.my] \\ ${ }^{2 *}$ School of Computer Science and Electronics Engineering, University of Essex, Colchester, CO4 3SQ, United \\ Kingdom \\ [e-mail: anisi@ieee.org, anisi@gmail.com] \\ *Corresponding authors: Rafidah Md Noor and Mohammad Hossein Anisi
}

Received February 6, 2017; revised May 15, 2017; accepted June 24, 2017;

published October 31, 2017

\begin{abstract}
Wireless signal transmission is influenced by environmental effects. These effects have also been challenging for Vehicular Ad hoc Network (VANET) in real-time communication. More specifically, in an urban environment, with high mobility among vehicles, a vehicle's status from the transmitter can instantly trigger from line of sight to non-line of sight, which may cause loss of real-time communication. In order to overcome this, a deterministic signal propagation model is required, which has less complexity and more feasibility of implementation. Hence, we propose a realistic path loss model which adopts ray tracing technique for VANET in a grid urban environment with less computational complexity. To evaluate the model, it is applied to a vehicular simulation scenario. The results obtained are compared with different path loss models in the same scenario based on path loss value and application layer performance analysis. The proposed path loss model provides higher loss value in $\mathrm{dB}$ compared to other models. Nevertheless, the performance of vehicle-vehicle communication, which is evaluated by the packet delivery ratio with different vehicle transmitter density verifies improvement in real-time vehicle-vehicle communication. In conclusion, we present a realistic path loss model that improves vehicle-vehicle wireless real-time communication in the grid urban environment.
\end{abstract}

Keywords: line of sight; non-line of sight; Path loss; Vehicular Ad hoc Networks 


\section{Introduction}

Mobile Ad hoc Network (MANET) [1] [2] [45] [46] [47] allows direct communication between vehicles. The benefit of MANET is that no centralized infrastructure is needed to support the communication between mobile devices. Adopting the idea of MANET in a vehicular environment enables sharing information between vehicles. This delivering method has a huge advantage, which is to provide safe and reliable transportation using Vehicular Ad hoc Network (VANET) [3] [4] [5] [38]. However, implementing wireless ad hoc communication in vehicular environment, more specifically on the grid urban environment, is too complex. Although, a huge amount of research is performed with barriers such as vehicles mobility and environmental effects on wireless signal transmission, however, a reliable wireless ad hoc communication in VANET still requires an advanced research for safer transportation.

VANETs have been successfully used in the development of a new generation of vehicular systems and applications. A basic ad hoc vehicle to vehicle communication enables a quick establishment of a flexible and simple infrastructure between vehicles in large scale network for a better system and applications on top layer of the stack. The effectiveness of VANET has created a huge demand to enhance safety and reliability of the existing Intelligent Transportation Systems (ITS). In an urban environment, VANET acts as one of the main potential layer of ITS to cooperate with higher level architectures like smart cities [6] [34] [42] [43] [44]. However, in urban environments, the performance of VANET application layer is affected by the wireless specifications and environmental effects. Wireless channel and radio waves that carry application layer requirements must be designed in such a way to consider encountering signal transmission with environmental effects. When improving vehicle to vehicle wireless transmission to help application layer, the radio propagation model is a promising field to overcome the related issues. The behaviour of a vehicle to vehicle wireless signal transmission is in fact reflecting the design of radio propagation models including of environmental effects. Several attempts to improve physical layer using path loss modelling have been proposed, but the majority of the models have complexity in modelling and difficulty of implementation.

The physical layer of wireless communication is always influenced by environmental effects, which directly affects the performance of the application layer [7] [36]. Therefore, establishing a scalable real-time communication with reasonable performance requires a realistic propagation model. However, expansion and consideration of a new model should not have the complexity and limitation of implementation which can be identified in some of the recent proposed models. This is more important in VANET in urban environments because large and small scale effects on signal transmission are not deniable. Therefore, in this work we propose a model with less complexity and limitation of implementation in case of environmental dependency of the model. The main selected effects for the development of new model are diffraction and reflection, which commonly occur in grid urban environments surrounded by buildings [8] [35]. These effects can be used in different techniques like Ray Tracing [9] [10] [11] to improve the performance of vehicle to vehicle communication via a deterministic model. Signal ray's specifications and signal propagation angles from surface of buildings are important elements in modelling reflection and diffraction based on the ray tracing technique. These elements help to model a realistic ray tracing path loss based on reflection and diffraction, which improves non-line of sight vehicle-to-vehicle communication in the grid urban environment. This model is designed based on large-scale effects propagation, integrated in JiST/SWANS vehicular simulator and tested in a Chicago map scenario with grid 
road pattern. Since very early VANET standards and protocol establishments, radio propagation models have been designed and proposed for specific VANET environments, as our proposed model that is designed for a specific grid urban environment like Chicago map scenario. The contributions of this research are as follows:

- Study and experiment the existing propagation models based on performance of real time communication (packet delivery ratio, the average end-to-end) with a different transmitter density of vehicles.

- Design a model based on signal ray tracing included of diffraction and reflection caused by objects (buildings) in the urban grid environment.

- Implement the proposed model using simulation tools.

- Evaluate and analyse the proposed model based on the performance of real time communication (packet delivery ration and average end-to-end) with a different transmitter density of vehicles.

The remaining of this paper is organized as follows. A comparison is made between recent literature and models in section 2. Then a generalized point of view of our design and implementation with mathematical path loss derivation of each scenario presented in section 3. Lastly, in section 4 and 5 we describe a simulated case study and discuss the results obtained to conclude improvement of vehicle to vehicle wireless communication.

\section{Related Work}

The demand to improve vehicular communication led to establish several wireless vehicular protocols and standards for VANET. Communication medium like Wireless Local Area Network (WLAN) and Worldwide Interoperability for Microwave Access (WiMAX) [12] have been strengthening vehicular wireless communication. Wireless standards like IEEE 802.11a, b and $\mathrm{p}$ are familiar communication standards that have recently been integrated into many of vehicular ad hoc communications. IEEE 802.11b allocates $20 \mathrm{MHz}$ of channel bandwidth and it is used for [19] [41]WLANs, functions on $5 \mathrm{GHz}$ band with 52 subcarriers of Orthogonal Frequency Division Multiplexing (OFDM). OFDM uses different modulation schemes, Binary Phase-Shift Keying (BPSK), Quadrature Phase Shift Keying (QPSK) and Quadrature Amplitude Modulation (QAM). Based on different modulation schemes which are possible to get up to $52 \mathrm{Mbps}$, but the realistic throughput of IEEE $802.11 \mathrm{~b}$ is expected to be 20 Mbps [13]. The IEEE 802.11p [14] [37] is a more advanced version of 802.11b, which allows exchanging data between vehicles for longer range. Although this standard runs on 5.8 $\mathrm{GHz}$ band and supports longer range of communication transmission up to 1 kilometre, but throughput is less than IEEE802.11b [13] [16]. This standard has been recently integrated in several platforms, for example Dedicated Short Range Communication (DSRC) [15].

Using wireless technology to allow communication between vehicles is fundamental to the development of applications like traffic congestion systems. These applications require scalable real time communication between vehicles. Signal transmission of vehicular ad hoc communication in urban environments is affected by vehicle mobility and environmental effects. In situations like this, vehicles may locate either in line of sight or non-line of sight from the transmitter. Therefore, improving non-line of sight signal transmission using deterministic propagation model came to the attention of researchers in the past few years. Researchers worked on two categories of radio propagation models which are deterministic and probabilistic models. The difference between these models comes from the way of calculating received signal power. Deterministic models calculate received signal power based 
on the actual properties of the environment [16] [40], meanwhile probabilistic model calculates variance of received signals that allows obtaining true received signal from a range of values [17][19].

In an attempt to path loss modelling presented in [18], both line of sight (LOS) and non-line of sight (NLOS) models are designed based on ray tracing techniques in an urban environment. This is a deterministic model that requires several parameters from the environment. The main parameter that is used in these models are distance and antenna's height. Both of the LOS and NLOS models are designed specifically for junctions in grid environments. These models were evaluated according to transmitting signal and its coverage. The results stated in [18] show improvement in transmission coverage, but the author did not evaluate the performance of data connectivity of receiving vehicles based on application layer. This is also noticeable point in an NLOS propagation situation stated by [19] [39]. In this literature [19] the author's focus is on the NLOS propagation situation in a grid urban environment and also the presented model is based on ray tracing technique to extract different NLOS path loss models for each vehicle. In each NLOS model, large scale effects such as diffraction and reflection between the transmitter and receiver are estimated, and the path loss is designed based on these signal paths to each receiving vehicle. Deterministic models are static and environmental dependent, therefore mobility of vehicles can simply change the radio propagation situation. The author in [20] used the NLOS path loss model presented in [19] to improve it with reflection ray tracing and electric reflected field. Calculating the number of rays along the parallel road and the phase part of the reflected rays are improved by the authors to model the electric reflected path loss model.

Due to the effect of environment on wireless signal transmission, defining the environmental properties is one of the fundamental stages of path loss modelling. As it can be seen in [18] path loss is only modelled for segmented roads, while [20] [19] is specifically modelled for grid environments surrounded by buildings. Simultaneously mobility is also one of the major causes of losing real time transmission between vehicles. Propagation status of vehicles may quickly change between line of sight, non-line of sight, near non-line of sight and out of sight. The path loss model is therefore required to be evaluated by one of the existing mobility models. Many of the current literatures [18] [19] do not involve mobility models in evaluating path loss modelling. The other important aspect of path loss modelling is the feasibility and mathematical simplicity of the path loss model. Deterministic models calculate path loss based on environmental details, such as distance [18]. So all these environmental values involved in path loss modelling, must be accessible to vehicles for path loss calculation. An overview of the recent literatures is shown in Table 1, and their proposed path loss models shown in Table 2. From the literature [18], [19], [20], it was observed that combination of effects is not used specifically for real-time urban grid environment in wireless vehicular ad hoc communication. Moreover, the existing propagation models are not fully addressing a combination of effects for the urban gird environment. Based on ray tracing techniques for such effects, it is simply possible to predict the most suitable signal path according to properties of the wireless signal. This allows prediction of signal and improvement of signal transmission to near non-line of sight and out of sight propagation situations. Therefore, to overcome these challenges, we introduce a realistic path loss model that improves vehicle-vehicle wireless real-time communication in a grid urban environment. 
Table 1. Summary of testbed specifications from [18], [19] and [20]

\begin{tabular}{|l|c|c|c|}
\hline Measurement study & {$[\mathbf{1 8 ]}$} & {$[19]$} & {$[20]$} \\
\hline \hline Spectrum & $5.9 \mathrm{GHz}$ & $2.4 \mathrm{GHz}$ & $\begin{array}{c}900 \mathrm{MHz} \\
1.8 / 5.9 \mathrm{GHz}\end{array}$ \\
\hline IEEE Standard & $802.11 \mathrm{p}$ & $802.11 \mathrm{~b} / \mathrm{g}$ & $802.11 \mathrm{p}$ \\
\hline Propagation Situation & $\begin{array}{c}\text { NoPATH/LOS } \\
\text { NLOS }\end{array}$ & LOS/NLOS & LOS/NLOS \\
\hline Propagation Model & Two Ray Ground & Two Ray Ground & Two Ray Ground \\
\hline Fading & - & Rice/Rayleigh & Rice/Rayleigh \\
\hline Bit rate (Mbps) & 3 & 2 & 3 \\
\hline Bandwidth & $10 \mathrm{MHz}$ & $20,22 \mathrm{MHz}$ & $10 \mathrm{MHz}$ \\
\hline Modulation & OFDM & DSSS,OFDM & OFDM \\
\hline Mobility & - & - & IDM-LC \\
\hline Routing protocol & AODV & AODV & AODV/LAR \\
\hline Simulation Tools & NCTUNS & QualNet/TIGER & GloMoSim \\
\hline
\end{tabular}

Table 2. LOS and NLOS models emptied in same literatures of Table 1

\begin{tabular}{|l|l|l|}
\hline \multirow{4}{*}[18]{} & LOS & LOS $=\{7.2+7.1 \log (\mathrm{ht} * \mathrm{hr} / \lambda)\} \log (\mathrm{d})+28.3 \log (1+\mathrm{d} / \mathrm{d} 0)-1.2 \log (\mathrm{f})-19.6 \log (\mathrm{WS})+65.9$ \\
\cline { 2 - 4 } & NLOS & $\begin{array}{l}\mathrm{NLOS} 2=\{47.6+6.6 \log (\mathrm{ht} \mathrm{hr} / \lambda)\} \log (\mathrm{d})+\{89.1-33(\mathrm{~d} 1 / \lambda)\} \\
\log (1+\mathrm{d} / \mathrm{db})+19.9 \log (\mathrm{f})-11.3 \log (\mathrm{W} 1 * \mathrm{~W} 2)+2.8\end{array}$ \\
\hline \multirow{2}{*}[19]{} & LOS & $\mathrm{PL}=20 \log (4 \pi \mathrm{r} / \lambda)$ \\
\cline { 2 - 4 } & NLOS & $\begin{array}{l}\mathrm{PL}(\mathrm{A})=10 \log (10 \mathrm{PL}(\mathrm{D}) / 10+10 \mathrm{PL}(\mathrm{R}) / 10) \\
\mathrm{PL}(\mathrm{B})=10 \log (10 \mathrm{PL}(\mathrm{DD}) / 10+10 \mathrm{PL}(\mathrm{RD}) / 10+10 \mathrm{PL}(\mathrm{DR}) / 10+10 \mathrm{PL}(\mathrm{R}) / 10)\end{array}$ \\
\hline \multirow{2}{*}[20]{} & LOS & $\mathrm{L}(\mathrm{r})=40.3+23.4 \log (\mathrm{r})$ \\
\cline { 2 - 4 } & NLOS & $\mathrm{L}=20 \log ((\lambda / 4 \pi)|\mathrm{Etot} / \mathrm{E} 0|)$ \\
\hline
\end{tabular}

\section{Path Loss Improvement and Modelling}

Ray tracing techniques are applicable for modelling the signal propagation in the grid urban environment. Therefore, it is possible to improve vehicle to vehicle communication using ray tracing technique and by combining large scale effects with a maximum possible transmission range of wireless standard. To do so we began with feasibility checking of path loss modelling based on road patterns and wireless maximum range of transmission. Then we identified and evaluated possible paths that signal may be propagated by combining the effects like diffraction and reflection towards the destination [20], [40].

In order to calculate a realistic path loss model in a grid urban environment, it is necessary to study the road patterns and the signal transmission range. Road patterns generate line of sight and non-line of sight propagation situations, and affects the functionality of the physical layer of transmission. In this experiment, roads are patterned with two intersections in respect to the vehicle's wireless transmission range. According to our real environment experiment, the highest possibility of occurrence of non-line of sight is road patterns intersecting with approximately $45^{\circ}$ to $90^{\circ}$. Realistically, transmission range decreases from transmitter to receiver due to the propagation effects caused by the breaking point of the roads. In case of a straight road, the transmission range is up to 180 meters, roads with one or two breaking points at the beginning and the end of road with $45^{\circ}$ range 130 to 140 meters, and grid roads with $90^{\circ}$ breaking point reduces the coverage to 120 meters. Path to destination in case of $90^{\circ}$ could be 
propagated via a combination of reflections and diffractions within 120 meters. According to Fig. 1 (b), the path loss model includes several possible paths between transmitter $T_{X}$ and receiver $R_{X}$, which we have derived from some possible reflection and diffraction signals in the grid urban environment. These paths may be transmitted with a reflected signal that is being diffracted to reach $\mathrm{R}_{\mathrm{X}}$, and vice versa. The most important parameters in this modelling, are to calculate the number of rays, diffraction points and signal reflection and diffraction degrees. Although signals may be transmitted to receiving vehicle in various paths, the paths that are proposed here are evaluated and selected according to simplicity with minimized rays, power loss and a combination of large scale effects. Basically signal attenuation is increased in each reflected, diffracted point and therefore not all possible paths may survive in an urban environment. So firstly we observed that the performance of real time communication is simply vulnerable to the type of roads specifically with grid patterns. Each specific pattern requires a specific signal propagation through a combination of the effects. Secondly, we obtained that it is feasible to rely on maximum wireless communication coverage, and use it to produce paths towards the destination with the combination of the effects using the ray tracing technique [36] [37].

The path loss is one of the main concepts of electromagnetic waves in radio signal propagation. In a general description, path loss is the power density reduction of electromagnetic waves while it propagates through space [21] [38] [39]. This verifies that the power of receiving signals on receiver $\mathrm{P}_{\mathrm{RX}}$ is less than the power of transmitting signal $\mathrm{P}_{\mathrm{TX}}$. According to equation (1), received power is calculated with the gain of the transmitting and receiving signal $\mathrm{G}_{\mathrm{TX}}$ and $\mathrm{G}_{\mathrm{RX}}$ as isotropic, and linear gain equals to 1 [21]. The relation between path loss and transmission power is the $\mathrm{dB}$ ratio of $\mathrm{P}_{\mathrm{TX}} / \mathrm{P}_{\mathrm{RX}}$. Therefore, the path loss in free space model is calculated in $\mathrm{dB}$ as shown in equation (2) [19].

$$
\begin{aligned}
& P_{R X}=P_{T X} G_{T X} G_{R X} \lambda^{2} / 16 \pi^{2} r^{2} \\
& 10 \log \left(\frac{P_{\mathrm{TX}}}{P_{R X}}\right)=10 \log \left(\frac{4^{2} \pi^{2} r^{2}}{\lambda^{2}}\right)=>P L=20 \log \left(\frac{4 \pi r}{\lambda}\right)
\end{aligned}
$$

Path loss in equation (2) is used to calculate loss of signal propagation. According to [20], [22] $\mathrm{PL}_{\mathrm{R}}$ (single reflection) is used to derive $\mathrm{PL}_{\mathrm{RR}}$ (double reflection) as equations (3) and (4), and $\mathrm{PL}_{\mathrm{D}}$ (single diffraction) stated as equations (5) and (6). These equations will then be used to model the path loss based on possible paths that were discussed in the previous section. In order to calculate the reflection it is required to know the minimum number of rays $\left(\mathrm{N}_{\min }\right)$ from the transmitting vehicle to the receiving vehicle [23] [40]. The value $r$ indicates the distance to intersections which is $r_{m}$ and $r_{s}$ in the case of reflection towards a single intersection, and $r_{m}, r_{s}$ and $r_{p}$ in case of double reflection signal paths. In the previous section we have identified a few possible signal paths from $T_{X}$ to $R_{X}$. Although there are a variety of possible paths to $R_{X}$, but our focus is to identify paths based on the combination of the effects like reflection and diffraction. According to Fig. 1 (b) there are five paths between $T_{X}$ and $R_{X}$, which can be mathematically modelled as equation (7). We have used LOS, NLOS and NLOS1 equations that are stated in [19] to develop path loss models for our suggested signal paths. The total path loss from $\mathrm{T}_{\mathrm{X}}$ to $\mathrm{R}_{\mathrm{X}}$ is equal to the sum of all possible paths including diffractions and reflections. Each path is separately modelled in the following section. These paths are based on vehicle movement towards the junctions and away from the transmitter, which is shown in Fig. 1 (a). 


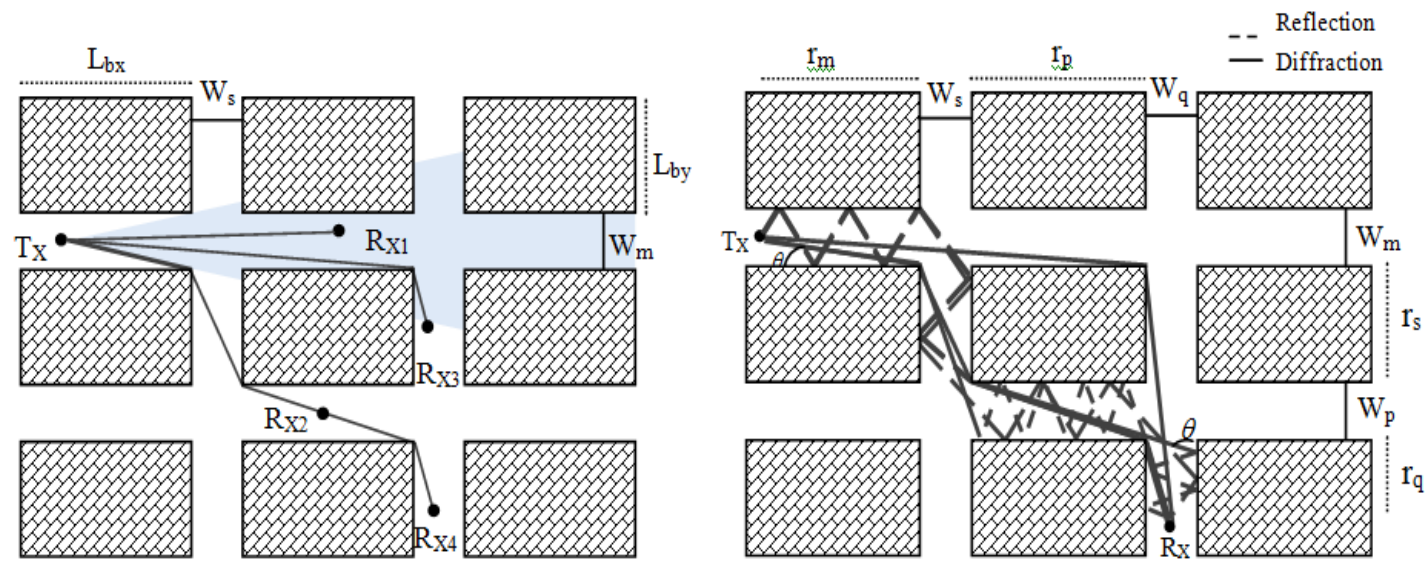

(a)

(b)

Fig. 1. (a) Graphical view of transmitting $T_{X}$ and receiving $R_{X}$ vehicles in different propagation situations (b) graphical view of the geometry of the proposed model

$$
\begin{aligned}
& \mathrm{PL}_{\mathrm{R}}=10 \log \left(\frac{\lambda}{4 \pi\left(\mathrm{r}_{\mathrm{m}}+\mathrm{r}_{\mathrm{s}}\right)}\right)^{2}+\mathrm{L}_{\mathrm{w}} \mathrm{N}_{\min } \\
& \mathrm{PL}_{\mathrm{RR}}=10 \log \left(\frac{\lambda}{4 \pi\left(\mathrm{r}_{\mathrm{tm}}+\mathrm{r}_{\mathrm{ts}} \mathrm{r}_{\mathrm{t}}\right)}\right)^{2}+\mathrm{L}_{\mathrm{w}} \mathrm{N}_{\min } \\
& \text { if }\left(\mathrm{r}_{\mathrm{M}}>\mathrm{f}_{\mathrm{S}}\right) \\
& \mathrm{PL}_{\mathrm{D}}=10 \log \left(\left(\frac{\lambda}{4 \pi \mathrm{r}_{\mathrm{m}}}\right)^{2}\right)+10 \log \left(\frac{\lambda \mathrm{r}_{\mathrm{m}}}{4 \mathrm{r}_{\mathrm{s}}{ }^{2}}\right) \\
& \text { if }\left(\mathrm{f}_{\mathrm{S}}>\mathrm{f}_{\mathrm{m}}\right) \\
& \mathrm{PL}_{\mathrm{D}}=10 \log \left(\left(\frac{\lambda}{4 \pi \mathrm{r}_{\mathrm{s}}}\right)^{2}\right)+10 \log \left(\frac{\lambda \mathrm{r}_{\mathrm{s}}}{4 \mathrm{r}_{\mathrm{m}}{ }^{2}}\right) \\
& \mathrm{PL}=10 \log \left(10^{\frac{\mathrm{PL}_{\mathrm{DDD}}}{10}}+10^{\frac{\mathrm{PL}_{\mathrm{DRD}}}{10}}+10^{\frac{\mathrm{PL}_{\mathrm{RDD}}}{10}}+10^{\frac{\mathrm{PL}_{\mathrm{RDR}}}{10}}+10^{\frac{\mathrm{PL}_{\mathrm{R}}}{10}}\right)
\end{aligned}
$$

Reflection is one of the common large scale effects that occurs in urban environments. It is more prevalent when the area is surrounded by buildings. A reflected signal with different number of rays may travel from the transmitter to the receiver. Fig. $\mathbf{1}$ shows two paths consisting of reflected signals that travel along the roads and intersections to reach $\mathrm{R}_{\mathrm{X}}$. In Fig 2 (b), we showed one of the possible paths caused only by reflection. The most important parameter to calculate this path loss is to estimate the number of reflected rays along the road. Therefore, using equation (8), the minimum number of reflected rays is calculated. In this equation, $\mathrm{W}$ is the width of the intersection and $\mathrm{r}_{\mathrm{m}}$ indicates the distance from the transmitter to the intersection, $r_{p}$ and $r_{s}$ are distance between two consecutive intersections, and $r_{q}$ is the distance from the last intersection to the receiver. Then the path loss between $T_{X}$ to $R_{X}$ is derived as in equation (9).

In this scenario considering diffraction as another candidate for signal path with single effect, signal may travel along two directions. Firstly, a path is made of three diffracted signals to reach $R_{X}$. Secondly, it is also possible that the $R_{X}$ is reached by a single diffracted signal from a different path. In order to derive this path loss equation, it is required to calculate $\mathrm{A}_{1}{ }^{2}$ and $\mathrm{A}_{2}{ }^{2}$ 
which are the values of the respective diffracted corner. According to Fig. 2 (b) the path to the $\mathrm{R}_{\mathrm{X}}$ is made up of three diffracted signals. In the same condition, a diffracted signal from the first corner is equal to the third diffraction. Meanwhile, it is possible that the path to the $R_{X}$ is consisting of two diffractions from another direction. In our modelling $r_{m}, r_{s}, r_{p}$ and $r_{q}$, are length of building blocks which may have different values, therefore $\mathrm{A}_{1}{ }^{2}$ and $\mathrm{A}_{2}{ }^{2}$ are calculated based on the values of $r$ with different index. We have derived $\mathrm{PL}_{\mathrm{DDD}}$ using equation (12) by conditioning length of building blocks. It is also possible to calculate $\mathrm{A}_{3}{ }^{2}$ for the third building block using equation (10) and (11). In this scenario, the $\mathrm{R}_{\mathrm{X}}$ is reachable by the single diffraction as it is shown in Fig. 2 (b). Equation (5) and (6) stated in [20] are also used in order to calculate the path loss with single diffraction.

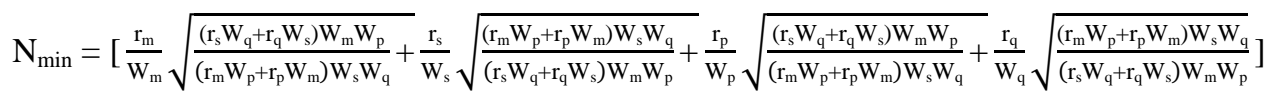

$$
\begin{aligned}
& \mathrm{PL}_{\mathrm{R}}=10 \log \left[\left(\frac{\lambda}{4 \pi}\right)^{2}\left(\frac{1}{\mathrm{r}_{\mathrm{m}}+\mathrm{r}_{\mathrm{s}}+\mathrm{r}_{\mathrm{p}}+\mathrm{r}_{\mathrm{q}}}\right)^{2}\right]+\mathrm{L}_{\mathrm{W}} \mathrm{N}_{\text {min }} \\
& \mathrm{A}_{1}^{2}=\frac{\lambda \mathrm{r}_{\mathrm{s}}}{4 \mathrm{r}_{\mathrm{m}} \mathrm{r}, \mathrm{m}} \quad \mathrm{r}_{\mathrm{m}, \mathrm{s}}<\mathrm{r}_{\mathrm{s}, \mathrm{m}} \\
& \mathrm{A}_{2}^{2}=\frac{\lambda \mathrm{r}_{\mathrm{p}}}{4 \mathrm{r}_{\mathrm{s}, \mathrm{s}}} \quad \mathrm{r}_{\mathrm{s}, \mathrm{p}}<\mathrm{r}_{\mathrm{p}, \mathrm{s}} \\
& \mathrm{PL}_{\mathrm{DDD}}=10 \log \left[\mathrm{A}_{1}^{2} \mathrm{~A}_{2}^{2}\left(\frac{\lambda}{4 \pi}\right)^{2} \frac{\lambda}{4 \mathrm{r}_{\mathrm{p}, \mathrm{q}} \mathrm{r}_{\mathrm{p}, \mathrm{p}}{ }^{2}}\right] \quad \mathrm{r}_{\mathrm{p}, \mathrm{q}}<\mathrm{r}_{\mathrm{q}, \mathrm{p}}
\end{aligned}
$$

In many of the recent literatures related to radio propagation, ray tracing technique with a combination of large scale effects such as diffraction and reflection is not used to expand NLOS propagating situations. In our model, we have expanded signal propagation to cover more out of sight and near non line of sight situations around vehicles based on possible, feasible shortest paths comprising a combination of effects. We have selected three of these paths that comprise a combination of the effects to derive the path loss model. These selected paths are based on wireless transmission range and environmental specification. The path loss models are mathematically modelled. Then two of these models with maximum combination of effects like reflection and diffraction are selected to be implemented in the Jist/SWANS simulator.

The first path is a signal reflection towards the first intersection is being diffracted to be transmitted to $\mathrm{R}_{\mathrm{X}}$. In order to model this path loss, a minimum number of reflected rays (Nmin) and values of diffraction points $\left(\mathrm{A}_{1}{ }^{2}\right.$ and $\left.\mathrm{A}_{2}{ }^{2}\right)$ are needed for calculation using the equations (13), (14) and (15). In this model, we assume that change of signal reflection to diffraction does not decrease the signal strength. Equation (16) is then used to calculate the path loss of the single reflection with double diffraction ( $\mathrm{PL}_{\mathrm{RDD}}$ ) shown in Fig. 2 (b).

$$
\begin{aligned}
& \mathrm{N}_{\min }=\left[2 \sqrt{\frac{\mathrm{r}_{\mathrm{m}} \mathrm{r}_{\mathrm{s}}}{\mathrm{W}_{\mathrm{m}} \mathrm{W}_{\mathrm{s}}}}\right] \\
& \mathrm{A}_{1}{ }^{2}=\frac{\lambda \mathrm{r}_{\mathrm{p}}}{4 \mathrm{r}_{\mathrm{s}} \mathrm{r}, \mathrm{s}} \quad \mathrm{r}_{\mathrm{s}, \mathrm{p}}<\mathrm{r}_{\mathrm{p}, \mathrm{s}} \\
& \mathrm{A}_{2}{ }^{2}=\left[\frac{\mathrm{r}_{\mathrm{s}}}{\mathrm{r}_{\mathrm{m}} \mathrm{r}_{\mathrm{s}}}\right]^{2} 10^{\frac{\mathrm{L}_{\mathrm{w}} \mathrm{N}_{\min }}{10}} \quad \mathrm{r}_{\mathrm{s}, \mathrm{p}}<\mathrm{r}_{\mathrm{p}, \mathrm{s}} \\
& \mathrm{r}_{\mathrm{s}, \mathrm{p}}<{ }_{\mathrm{rp}, \mathrm{s}}, \mathrm{r}_{\mathrm{p}, \mathrm{q}}<\mathrm{r}_{\mathrm{q}, \mathrm{p}} \\
& \mathrm{PL}_{\mathrm{RDD}}=10 \log \left[\mathrm{A}_{1}{ }^{2} \mathrm{~A}_{2}{ }^{2}\left(\frac{\lambda}{4 \pi}\right)^{2} \frac{\lambda}{4 \mathrm{r}_{\mathrm{p}, \mathrm{q}} \mathrm{r}_{\mathrm{q}, \mathrm{p}}{ }^{2}}\right]+\mathrm{L}_{\mathrm{W}} \mathrm{N}_{\min }
\end{aligned}
$$


The second path has similar reflection and diffraction occurrence, but the signal is lastly reflected to $\mathrm{R}_{\mathrm{X}}$. Using the second path in this scenario shown in Fig. 2 (b), the reflection angel $\theta$ has to be known in order to calculate the number of rays $(\mathrm{N})$ for the second reflection. Since $\theta$ is hard to find, we use a different equation (equation 17) which uses more accessible values to calculate the number of rays. The value of $\tan \theta$ in equation 17 can be identified by dividing the paths of reflection over the paths of diffraction. Then equation (18) is used to calculate the path loss of double reflection and single diffraction ( $\left(\mathrm{L}_{\mathrm{RDR}}\right)$ shown in Fig. 2 (a).

$$
\begin{gathered}
\mathrm{N}=\left[\frac{\mathrm{r}_{\mathrm{q}}}{\mathrm{W}_{\mathrm{q}}} \tan \theta\right]=\left[\frac{\mathrm{r}_{\mathrm{q}} \mathrm{r}_{\mathrm{p}}}{\mathrm{W}_{\mathrm{q}} \mathrm{W}_{\mathrm{p}}}\right] \\
\mathrm{PL}_{\mathrm{RDR}}=10 \log \left[\mathrm{A}_{1}^{2} \mathrm{~A}_{2}{ }^{2}\left(\frac{\lambda}{4 \pi r_{\mathrm{q}}^{2}}\right)^{2}\right]+\mathrm{L}_{\mathrm{W}} \mathrm{N}_{\min }+\mathrm{L}_{\mathrm{W}} \mathrm{N}
\end{gathered}
$$

Lastly, the third path towards receiver $\mathrm{R}_{\mathrm{X}}$ may consist of double diffraction and single reflection but in different sequences. Assuming signal diffraction occurs at the first intersection and being reflected towards the second intersection and then reflected to $\mathrm{R}_{\mathrm{X}}$. In Fig. 2 (b) diffracted signal is reflected towards the road and hits the corner of building blocks to reach $\mathrm{R}_{\mathrm{X}}$ as a double diffracted signal. Path loss of double diffraction and single reflection $\left(\mathrm{PL}_{\mathrm{DRD}}\right)$ in this scenario is then calculated using equation (19). Note that equation (19) is derived from the combination of equations (14) and (17).

$$
\mathrm{PL}_{\mathrm{DRD}}=10 \log \left[\mathrm{A}_{1}^{2}\left(\frac{\lambda}{4 \pi}\right)^{2} \frac{\lambda}{4 \mathrm{r}_{\mathrm{r}, \mathrm{q}} \mathrm{r}, \mathrm{p}} \mathrm{p}\right]+\mathrm{L}_{\mathrm{W}} \mathrm{N}
$$

\section{Feasibility and implementation}

We aimed at two approaches with conducting a simulated case study. Firstly evaluation of physical layer was required to establish the feasibility of our theory. Secondly, to analyse the performance and to evaluate accuracy of data transmission regarding different path loss models with different transmitter density in the grid urban environment.

In order to analyse the properties of a physical layer, VisSim Comm [24] is selected to model and simulate IEEE 802.11p [25] wireless spectrum. This tool helps to simulate end-to-end communication at the physical layer in the form of block diagrams. With respect to demand of wireless signal range, OFDM subcarriers are set to BPSK modulation [26]. The first optional stage of the data transmission is convolutional encoding, which improves the error probability. Meanwhile, we needed to find a suitable urban environment with grid streets surrounded by buildings to evaluate performance of scenario configured with the proposed path loss model. JiST/SWANS [27] [28] is a simulation tool that we used for vehicle to vehicle application layer performance evaluation based on the proposed model [35]. It is well designed for vehicular simulation which supports IEEE $802.11 \mathrm{p}$ standard and mobility models. This simulator includes a Chicago map which is a suitable environment to evaluate the performance of data transmission in the grid urban environment for our proposed path loss model. This simulated environment is in a grid urban environment which is surrounded by tall buildings. The environmental configuration is done via an XML file. In this simulated environment we have set different densities of vehicles as well as different densities of transmitting vehicles. Our target is to evaluate metrics such as packet delivery ratio with proposed path loss models. Then we verify the efficiency of the proposed model through comparison of performance metrics with Two Ray Ground, Free Space and Shadowing models. 


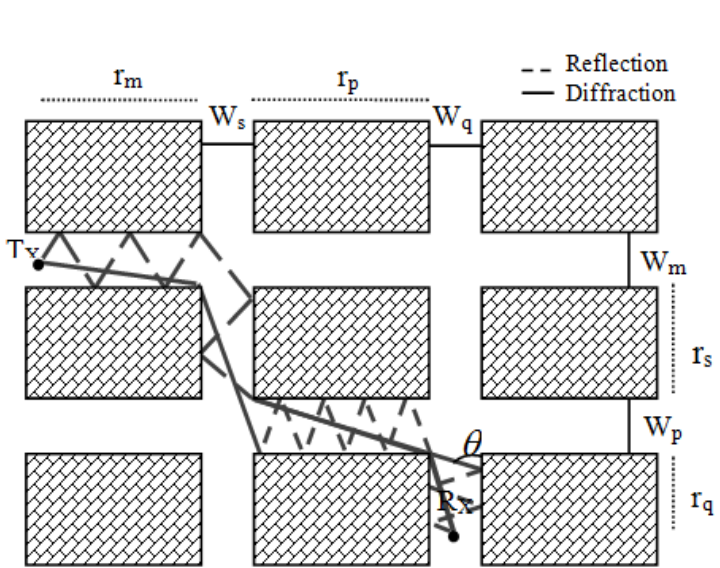

(a)

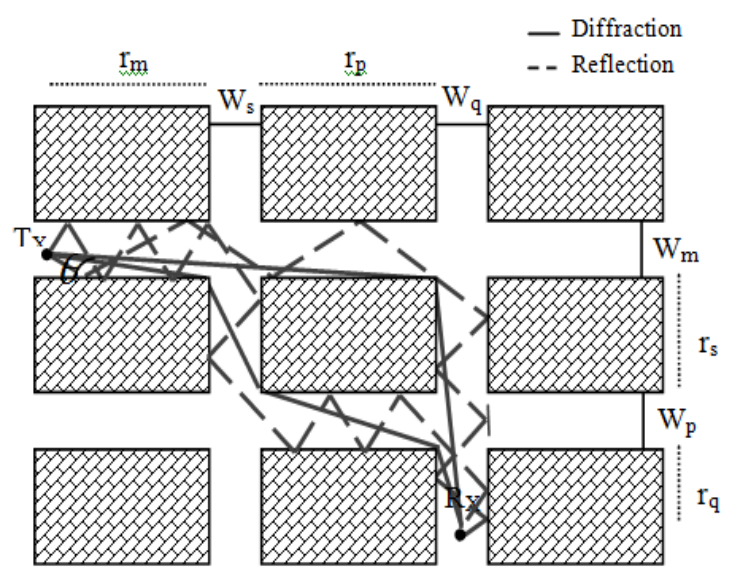

(b)

Fig. 2. (a) Illustration of signal path from transmitter $T_{X}$ to receiver $R_{X}$ by single effect, (b) Illustration of signal path from transmitter $T_{X}$ to receiver $R_{X}$ by combination of the effects

Free Space model is also called Friis model [44]. In this model, transmission depends on transmission power, antenna gain and distance between transmitter to receiver. Using this model, it is expected to have a single direct signal path between transmitter and receiver. Two Ray Ground model is a more advance version of the Free Space model where received signal power is calculated based on a single direct path and also a ground reflected path. Height of the antenna is also one of the parameters for calculating received signal power using Two Ray Ground model and shadowing model. These models provide better transmission in comparison with Free Space Model [16] [19].

We have set up a simulated environment with the specifications listed in Table 3. Using the XML file we configured 100 vehicles with the Random Waypoint mobility a model with the grid urban environment. Vehicles communicate directly to each other using Ad hoc on Demand Distance Vector (AODV) [29] as routing protocol. Wireless communication is configured to IEEE $802.11 \mathrm{~b}$. Table 4 presents wireless standards that have been commonly used in either real environments or simulated wireless vehicular studies. Rice and Rayleigh fading are also optionally included in transmission channel as well as path loss.

Signal strength is an important issue when the path is dealing with a combination of large scale of the effects. As mentioned earlier, a signal looses power once reflection or diffraction occurs. Also signal looses power when it changes from diffraction to reflection and vice versa. Other issues such as fading and shadowing also occur in urban environments, and cause attenuation. These parameters can be configured in the environmental parameters in an XML file. In our project, loss in the XML file is set to 0 , and the scenario is only applies to path loss. We have also configured the $2.4 \mathrm{GHz}$ wireless spectrum, which together with the $5.8 \mathrm{GHz}$ spectrum was tested in the case of power $\mathrm{dB}$ in the VisSim Comm simulator. The power density of 2.4 GHz spectrum is from 12 to 25 and 30 to $45 \mathrm{~dB}$ meanwhile $5.8 \mathrm{GHz}$ spectrum has a power density distribution from 20 to $50 \mathrm{~dB}$. In terms of power density, $5.8 \mathrm{GHz}$ could also be used in our experiment. However, our proposed models are designed for short distance communication with higher power density distribution in vehicular networks. Other involved parameters such as mobility, node specifications, protocols and map specifications are configured in the XML file 
Table 3. JIST specifications used in our experiment

\begin{tabular}{|l|c|}
\hline Parameters & Value \\
\hline \hline Simulation tools & JIST \\
\hline Number of vehicles & 100 \\
\hline Simulation time & 400 seconds \\
\hline Simulation map & XML, Chicago map \\
\hline PHY standard & 802.11b \\
\hline $\begin{array}{l}\text { Radio propagation } \\
\text { model }\end{array}$ & $\begin{array}{c}\text { Two Ray Ground, Free } \\
\text { Space, Shadowing }\end{array}$ \\
\hline Mobility model & \begin{tabular}{c} 
Random Waypoint \\
\hline Routing protocol
\end{tabular} \\
\hline
\end{tabular}

Table 4. Commonly used IEEE standards in VANET [14]

\begin{tabular}{|l|c|c|c|}
\hline IEEE Standard & $\mathbf{8 0 2 . 1 1 a}$ & $\mathbf{8 0 2 . 1 1 b}$ & $\mathbf{8 0 2 . 1 6}$ \\
\hline \hline Release Date & $1999 / 2001$ & 2007 & 2001 \\
\hline $\begin{array}{l}\text { Frequency Range } \\
\text { MHz }\end{array}$ & $5-6 \mathrm{GHz}$ & $\begin{array}{c}5855-5875 \\
5875-5905 \\
5905-5925\end{array}$ & $10-66 \mathrm{GHz}$ \\
\hline Channel & $20 \mathrm{MHz}$ & $5,10,20 \mathrm{MHz}$ & $20,25,28 \mathrm{Mhz}$ \\
\hline Modulation & BPSK/QPSK & $\begin{array}{c}\text { BPSK/QPSK } \\
16 \mathrm{QMM} / 64 \mathrm{QAM}\end{array}$ & $\begin{array}{c}\text { QPSK/16QAM } \\
64 \mathrm{Q} \text { AM } / 64 \mathrm{Q}\end{array}$ \\
\hline Data rate & $54 \mathrm{Mbps}$ & $5.8 \mathrm{Mbps}$ & $32-134 \mathrm{Mbps}$ \\
\hline Range (Indoor) & $0.035 \mathrm{Km}$ & - & - \\
\hline Range (Outdoor) & $0.12 \mathrm{Km}$ & $1 \mathrm{Km}$ & $4-5 \mathrm{Km}$ \\
\hline
\end{tabular}

\subsection{Simulation Experiments}

The implementation begins with configuring a XML scenario in Jist/SWANS, and then mathematical formula of path loss that is modeled and integrated in java code in Jist/SWANS A suitable environment is the main concern of experimenting radio propagation. Propagation models are designed for specific environment and conditions. The model is proposed in this article focus on NLOS propagation situation, therefore grid urban environment is a proper scenario to challenge the model. This grid urban environment is surrounded by tall buildings. Buildings are the major cause of signal reflection and diffraction and increase occurrence of large scale effects. In Jist/SWANS simulation the grid urban environment is coded in XML language. Fig. 3 illustrates the simulated environment. The XML code is used to specify several parameters like mobility, node and environment options. The focus of this research is the environment options which allow changing path loss model.

The XML script is used to define the Chicago scenario. In this code, there are few elements that need to be specified. Street options, mobility options, node options.

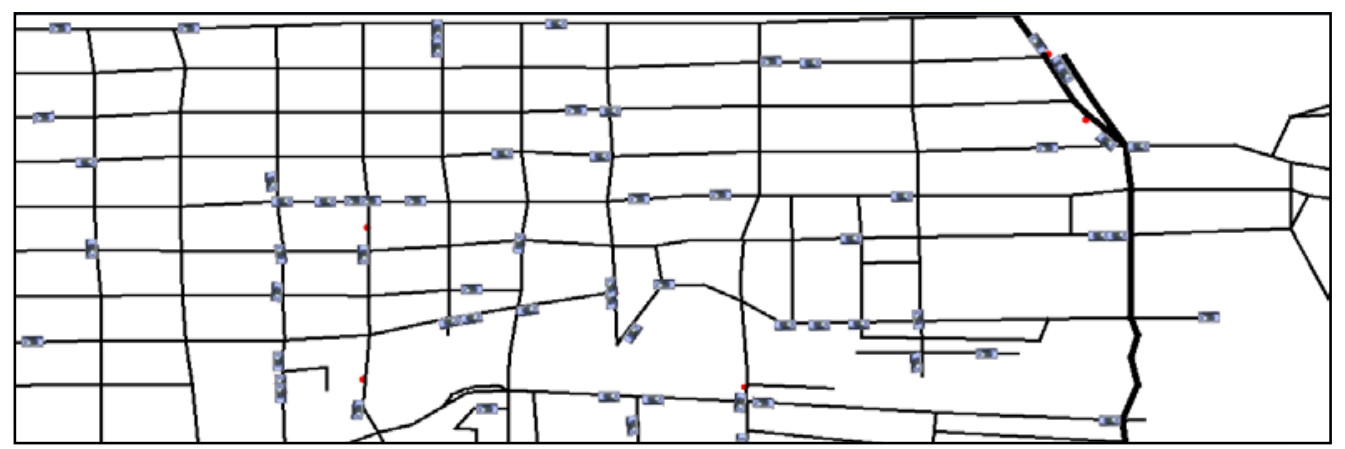

Fig. 3. Graphical view of simulated Chicago map 
The main reason is that combination of large scale effects such as reflection and diffraction were not examined in existing literatures. So implementation of these two models, $\mathrm{PL}_{\mathrm{DRD}}$ and $\mathrm{PL}_{\mathrm{RDR}}$ are presented. Simulation specifications such as wireless communication standard, mobility model, routing protocols and physical level must be carefully valued because of the direct effect on performance. There are some common IEEE standards that have been recently used in vehicular communication. VANET standards and specifications are listed in Table 4. In our experiment, vehicles are equipped with wireless ad hoc that supports IEEE 802.11b. This standard has been widely used in many of new ITS applications such as notification and warning systems. Vehicles are communicating directly to each other via Ad hoc protocol, and Ad hoc on Demand Distance Vector (AODV) is set as routing protocol [29].

\section{Results and Discussion}

We have implemented our proposed model in the JiST/SWANS simulator. According to the specifications of the simulated environment, we have collected value of path loss with proposed path loss model PL $L_{\text {DRD }}$ as well as Two Ray Ground, Free Space and Shadowing models. In addition, the simulation runs for 400 seconds in-order to achieve a quick response like real time processing system without significant delay. At present, Two Rag Ground and Free Space models are the most widely used models in VANET simulations. It is thus credible to be used in a tangible measurement with our proposed model. Based on collected values, we observed that range of path loss obtained from our model is similar to the work presented in [20] for a distance of 100-200 meters. Fig. 4 presents that our proposed path loss model provides a higher path loss compared to the three other models. The combination of the effects, more the number of rays, and signal power attenuation (because of the effects such as diffraction and reflection) cause a higher path loss in the proposed model compared to other models [23]. In Two Ray Ground and Free Space models with same vehicle transmitter density, minimum number of rays are propagated; therefore, path loss obtained from these models is lower than the proposed model. Approximate value of -82 to $-86 \mathrm{~dB}$ is expected for 20 meter Free Space model signal propagation [30]. However, according to Fig. 4, range of -90 to $-150 \mathrm{~dB}$ obtained with our model. A variation of the path loss value obtained from our model refers to the occurrence of combined effects (diffraction and reflection). Using our model, which is an improvement of the model presented in [22] and [23], IEEE 802.11b signal power of $20 \mathrm{dBm}$ travels an applicable distance to rely on for modelling path loss with antenna gain of 1 isotropic idB and antenna's height of 1 meter. With our proposed model, each time edge diffraction gives $-6 \mathrm{~dB}$ loss while according to the results stated in [23], the loss value obtained for single diffraction is $-2 \mathrm{~dB}$. We obtained $-15.174 \mathrm{~dB}$ power loss from the transmitter to the first corner (first time diffraction), for a $2.4 \mathrm{GHz}$ spectrum (received power for distance of 20 meters). We believe that the antenna gain of $7 \mathrm{idB}$ used by [23] provides less loss caused by diffraction because the transmitter power of $20 \mathrm{dBm}$ also used. Hence, although the path loss value of our model is higher than the path loss of the selected models, we decided to observe the effect of the proposed model on the performance of the application layer. It is expected that the signal propagation expansion of non line of sight and near non line of sight can improve the performance of vehicle-to-vehicle communication in the grid urban environment by a combination of the effects. 


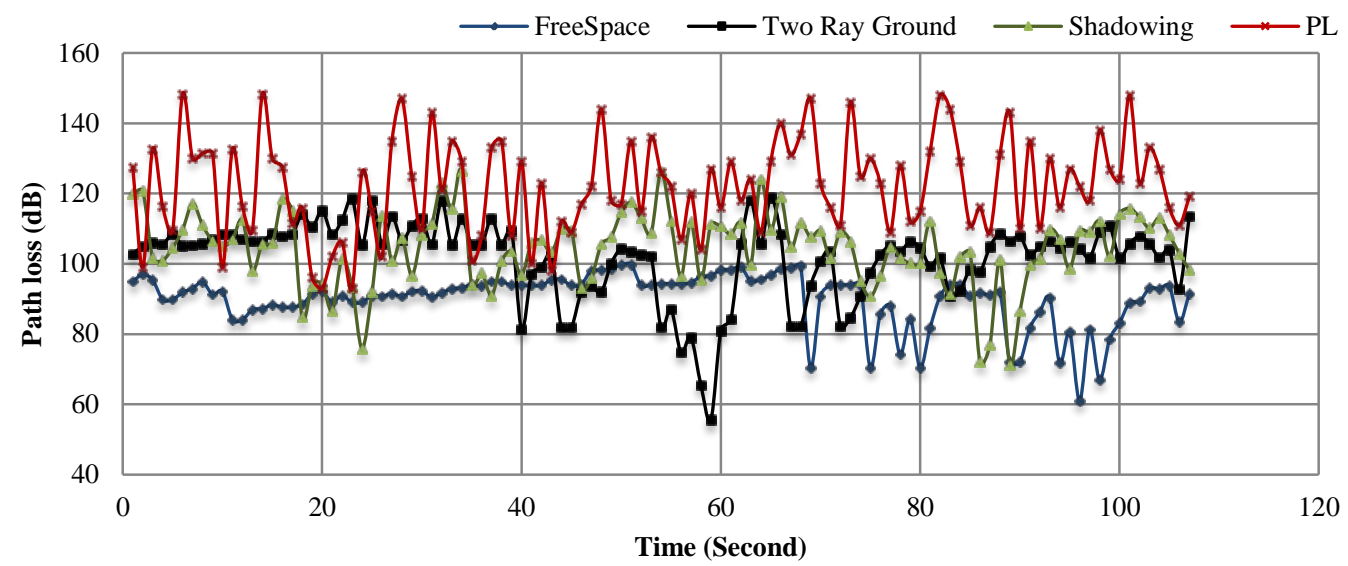

Fig. 4. Path loss values collected from the simulator with Two Ray Ground, Free Space, Shadowing and proposed model $\left(\mathrm{PL}_{\mathrm{DRD}}\right)$

In this work, after path loss evaluation, we observed performance of vehicle-vehicle communication using the packet delivery ratio. The packet delivery ratio is one of the important performance metrics that shows the accuracy of data transmission. Therefore, in this experiment, three scenarios are defined with 100 vehicles with a transmitting density of 0.1 , 0.25 , and 0.5. These scenarios are examined with Free Space, Two Ray Ground and Shadowing path loss [31] to make a comparison with proposed PL $\mathrm{DRD}_{\mathrm{DR}}$ and $\mathrm{PL}_{\mathrm{RDR}}$ models. Free space and Two Ray Ground are two common propagation models that have been recently used in a few literatures [31], [16] and [19]. With the expansion of the NLOS propagation situation around vehicles, it is expected that connectivity among vehicles increases. Although many vehicles may locate on near non line of sight propagation situation, they are in the signal transmitting range, but cannot receive signals. In contrast, using of our path loss model, it is expected to have better performance in data delivery. As Fig. 5 (a) shows the improvement of packet delivery ratio of applied $\mathrm{PL}_{\mathrm{DRD}}$ and $\mathrm{PL}_{\mathrm{RDR}}$ in comparison with Two Ray Ground, Free Space and Shadowing models.
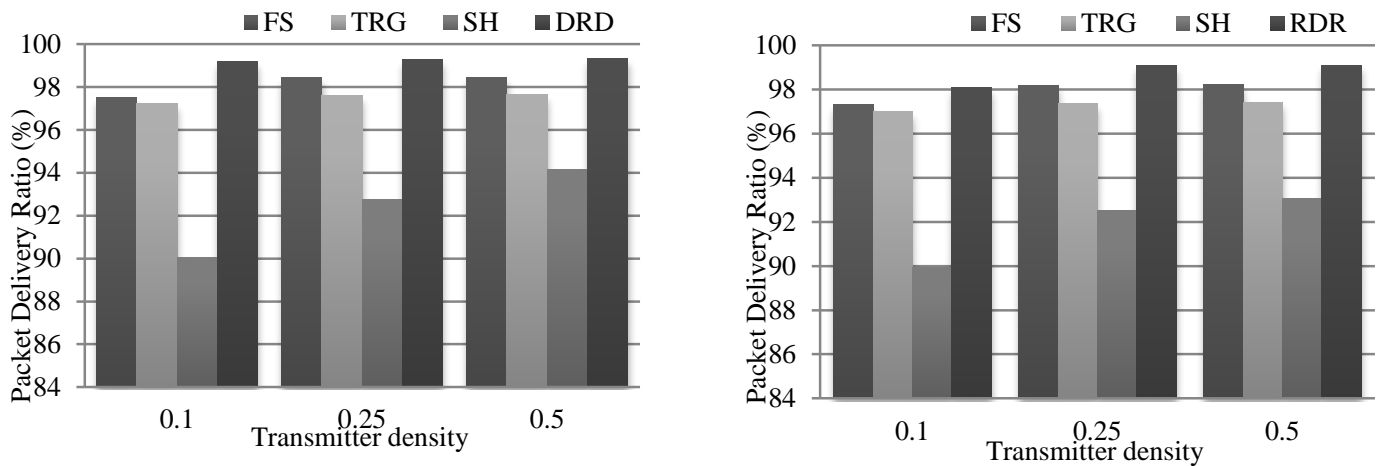

Fig. 5. (a). Packet delivery ratio comparison of Free Space (FS), Two Ray Ground (TRG), Shadowing $(\mathrm{SH})$, and $\mathrm{PL}_{\mathrm{DRD}}$ and $\mathrm{PL}_{\mathrm{RDR}}$ path loss models

Among these four path loss models, shadowing model with the lowest transmission among vehicles, on $\mathrm{PL}_{\mathrm{DRD}}$ with highest transmission is identified as the less and most suitable models. With less number of vehicles communicating, the signal travel less and chance of reaching the receiver is less, but in general signal may be blocked by the same objects on the street. In case 
of $\mathrm{PL}_{\mathrm{DRD}}$ firstly signal is predicted to travel from several paths to reach the receiver. This is the advantage over other models.

Average end to end delay is also one of the methods to evaluate performance of scenario. This method is to find out the average delay time of data delivery to the receiver. Fig 5. (b) presents average end to end delay time that obtained from the simulation experiment. In this research, the average end to end delay is tested with Free Space, Two Ray Ground, Shadowing, and proposed $\mathrm{PL}_{\mathrm{DRD}}$ and $\mathrm{PL}_{\mathrm{RDR}}$.
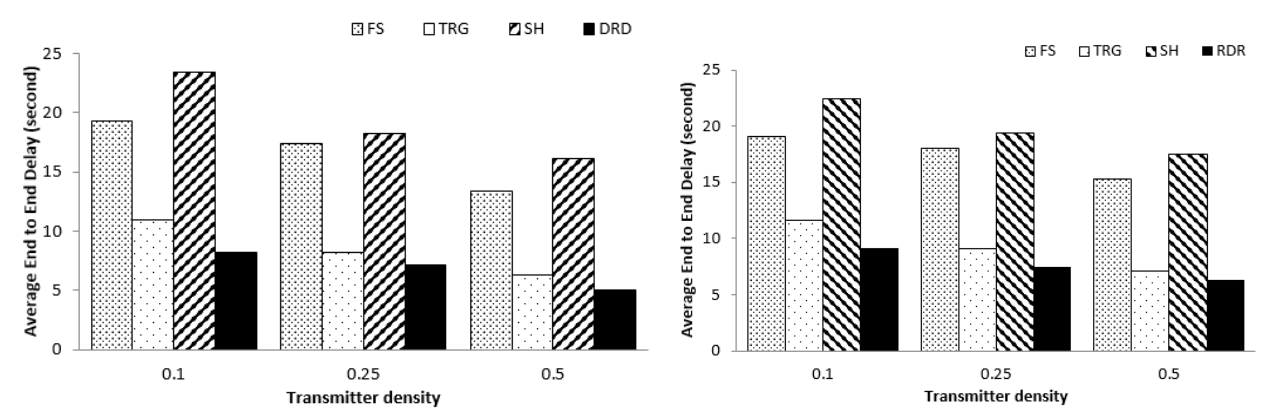

Fig. 5. (b) Average end to end delay comparison of Free Space (FS), Two Ray Ground (TRG), Shadowing (SH), $\mathrm{PL}_{\mathrm{DRD}}$ path loss model and $\mathrm{PL}_{\mathrm{RDR}}$ path loss model.

Average end to end delay (Fig. 5 (b)) and packet overhead (Figs. 4 (c)) both are exaggerated because of changing of model, it is also because of the High or Low density of vehicles and network. May also be a cause of delay in communication between vehicles [20].
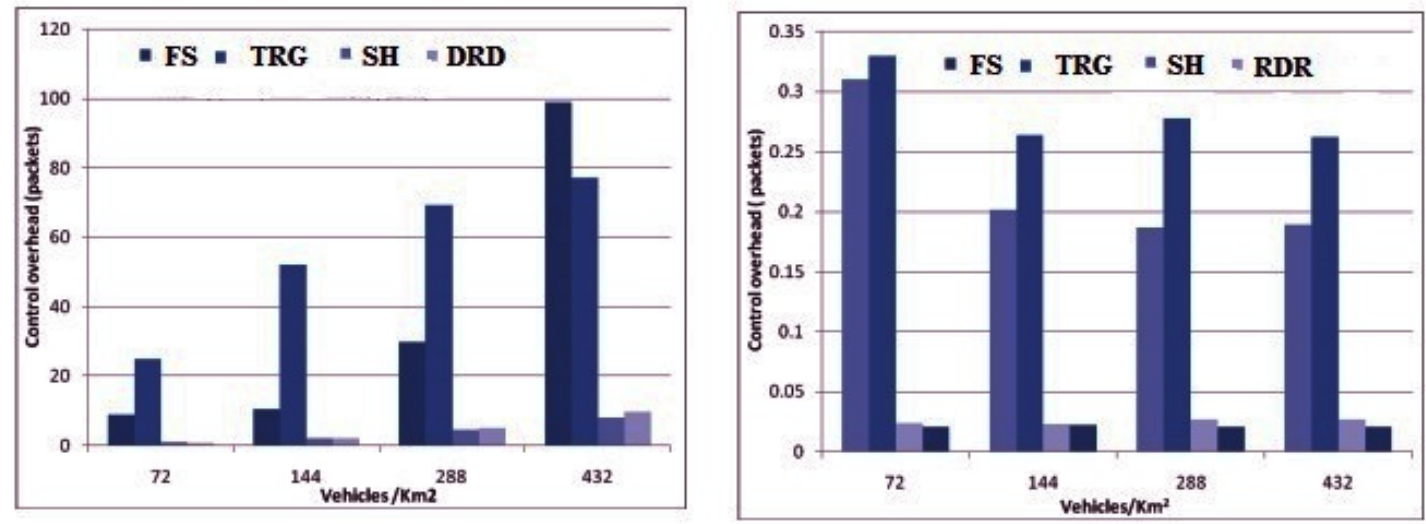

Fig. 5. (c) Control overhead comparison of Free Space (FS), Two Ray Ground (TRG), Shadowing (SH), $\mathrm{PL}_{\mathrm{DRD}}$ path loss model and $\mathrm{PL}_{\mathrm{RDR}}$ path loss model.

Fig. 6 (a) to Fig. 6 (d) shows the difference of connectivity between vehicles regarding to different path loss models. According to these figures, the red circles on the roads indicate communication between vehicles. The difference in density of red circles shows that in which scenario vehicle could communicate easier. We have taken snapshots of the Chicago map while wireless vehicular ad hoc communication is applied with Free Space, Two Ray Ground and Shadowing path loss models as well as our proposed path loss model. Testing communication density over the streets is one of the ways to evaluate NLOS propagation models. 


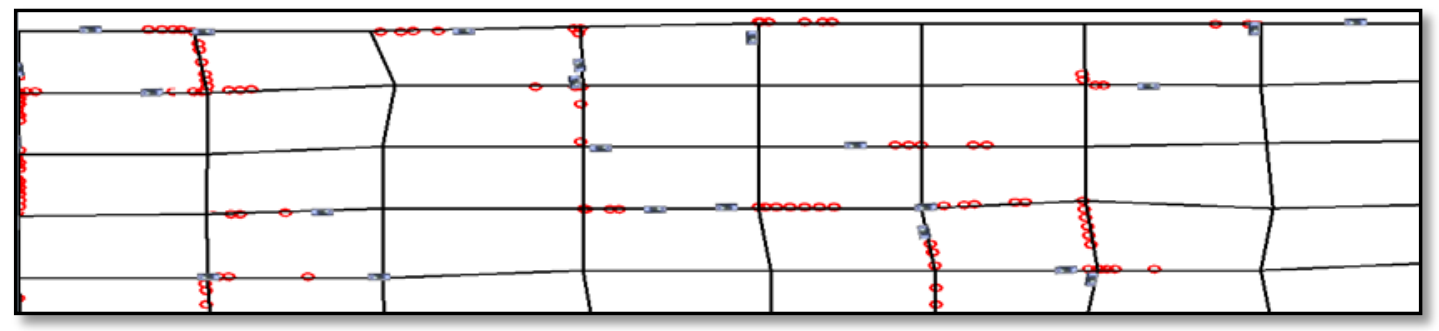

Fig. 5. (a) Snapshot of scenario from Jist/SWAN applied with Two Ray Ground path loss

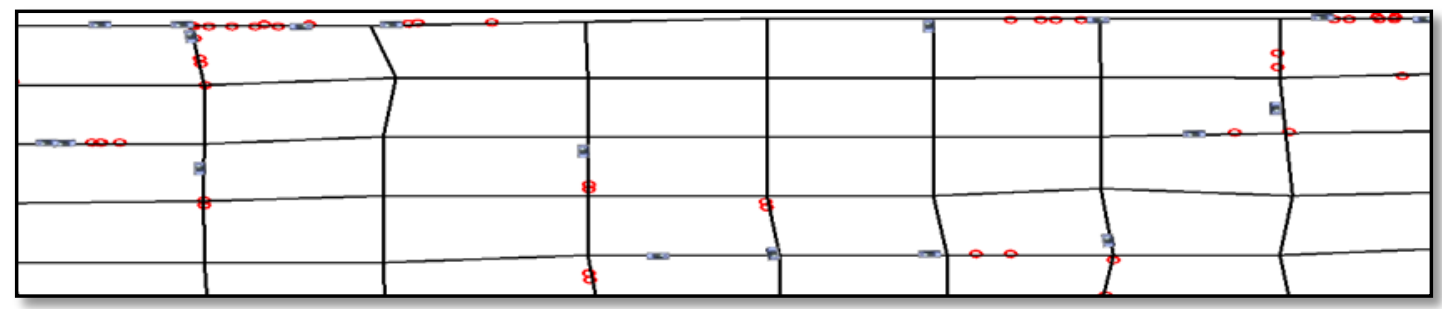

Fig. 6. (b) Snapshot of scenario from Jist/SWAN applied with Shadowing path loss

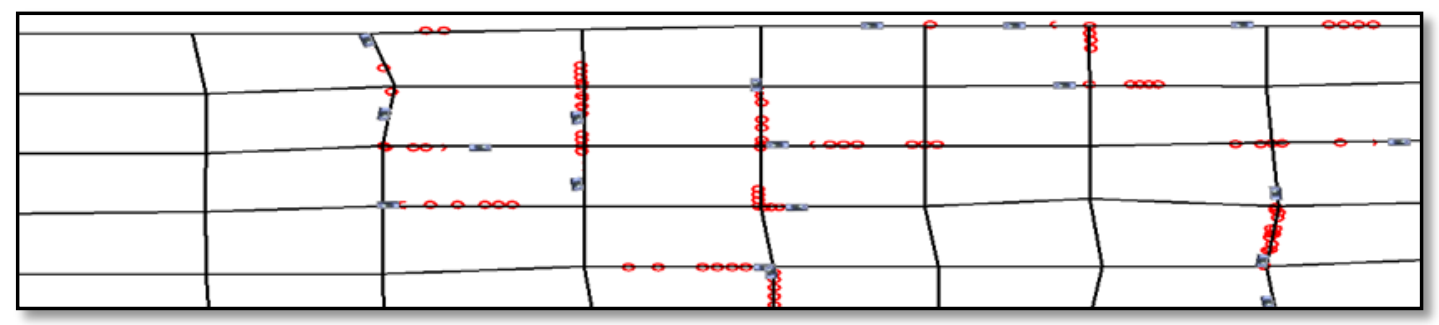

Fig. 6. (c) Snapshot of scenario from Jist/SWAN applied with Free Space path loss

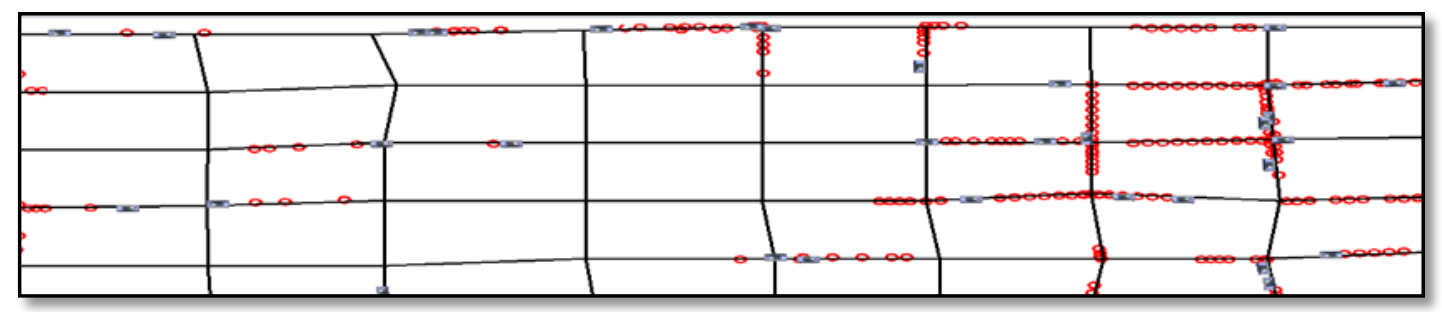

Fig. 6. (d) Snapshot of scenario from Jist/SWAN applied with proposed model PL $L_{D R D}$

It also can be seen in some recent researches that communication density is checked to identify the improvement of NLOS communication. The number of vehicles in a specific area with a higher density of red communication indicators along the street verifies the result provided in Fig. 6 (b) and Fig. 6 (c). Higher packet delivery ratio supports better communication density. Among these four path loss models, shadowing model with the lowest transmission among vehicles, on $\mathrm{PL}_{\mathrm{DRD}}$ with highest transmission is identified as the less and most suitable models. With less number of vehicles communicating, the signal is less travelled and chance of reaching the receiver is less, but in general signal may be blocked by the same objects on the street. In case of $\mathrm{PL}_{\mathrm{DRD}}$ firstly signal is predicted to travel from several paths to reach the receiver. This is the first advantage over other models. Two Ray Ground model predicts a 
direct LOS path and a ground reflected path to the receiver while free space model provides only a direct LOS path to the receiver. Basically, in-order to improve NLOS communication in urban environment, it is required to predict expanded area signal rays which aimed in this project. Also in this project combination of effects is targeted to improve vehicle-vehicle communication. So this is also a reason to verify the higher communication density according to Fig. 6 (d).

\section{Conclusion}

In this paper we design and implement a realistic path loss model in an urban grid environment for VANET. Using the JiST/SWANS simulator, we created a suitable environment to extract credible results with the applied path loss model. We show that a combination of large scale effects can be used in ray tracing techniques to develop a realistic path loss model. In addition, we design a path loss model with less mathematical complexity. In ray tracing techniques, path loss modelling depends on values from the environment. Instead in our modelling, these values are simplified to be more accessible for calculating path loss. Although the path loss value collected from the proposed model is higher than path loss of existing models, but the effect of the proposed model is verified by performance evaluation of the application layer. Expanding signal propagation of non line of sight and near non line of sight by a combination of the effects, results a significant improvement in performance of vehicle to vehicle communication in the grid urban environment. Moreover, In order to extend this research, this method can be implemented using IEEE 802.11p which provide longer range of transmission.

\section{References}

[1] J. Loo, J. L. Mauri, J. H. Ortiz, Mobile ad hoc networks: current status and future trends, CRC Press, 2016

[2] P. Rohal, R. Dahiya, P. Dahiya, "Study and Analysis of Throughput, Delay and Packet Delivery Ratio in Manet for Topology based Routing Protocols (AODV, DSR and DSDV),” Int. Journal for Advance Research in Engineering and Technology, vol. 1, no. 11, March 2013. Article (CrossRef Link)

[3] H. Moustafa, Y. Zhang, Vehicular Networks: Techniques, Standards, and Applications. USA, Auerbach Publications Boston, 2009.

[4] R. Michoud, A. M. Orozco, G. Llano, "Mobile Ad-Hoc Routing Protocols Survey for the Design of VANET Applications," in Proc. of the Intelligent Transportation Systems Symposium (CITSS) Colombia, 2012. Article (CrossRef Link)

[5] W. Chen, Vehicular communications and networks: Architectures, protocols, operation and deployment, Elsevier, 2015.

[6] A. Devare, A. Hande, A. Jha, S. Sanap, S. Gawade, "A survey on Internet of Things for Smart Vehicles," Int. Journal of Innovative Research in Science, Engineering and Technology, vol. 5, no. 2, pp. 1212-1217, February 2016. Article (CrossRef Link)

[7] P. Ren, J. Qian, "A Power-Efficient Clustering Protocol for Coal Mine Face Monitoring with Wireless Sensor Networks Under Channel Fading Conditions,” Sensors, vol. 16, no. 6, 2016. Article (CrossRef Link)

[8] L. U. Aguiar, C. T. Barba, J. E. Jiménez, M. A. Igartua, "On the Impact of Building Attenuation Models in VANET Simulations of Urban Scenarios,” Electronics vol. 4, pp. 37-58, 2015. Article (CrossRef link)

[9] V. D. Khairnar, K. Kotecha, "Propagation Models for V2V Communication in Vehicular Ad-hoc Networks," Journal of Theoretical and Applied Information Technology, vol. 61, no. 3, pp. 686-696, 2014. Article (CrossRef link) 
[10] V. S. S. Bohacek, G. Singh, A. Ilic, "The UDel Models: MANET Mobility and Path Loss in an Urban/Suburban Environment," University of Delaware, Electrical Engineering Department, Tech. Rep., 2004.

[11] E. Masson, Y. Cocheril, P. Combeau, L. Aveneau, M. Berbineau, R. Vauzelle, E. Fayt, "Radio wave propagation in curved rectangular tunnels at $5.8 \mathrm{GHz}$ for metro applications, simulations and measurements," EURASIP Journal on Wireless Communications and Networking, vol. 1, pp. 1-8, 2011. Article (CrossRef Link)

[12] J. M. Westall, J. J. Martin, "Performance Characteristics of an Operational WiMAX Network," IEEE Transactions on Mobile Computing, IEEE Educational Activities Department, vol. 10, no. 7, July 2011. Article (CrossRef Link)

[13] J. Jansons, A. Barancevs, E. Petersons, N. Bogdanovs, "IEEE802.11a Standard Performance in Mobile Environment," Int. Journal On New Computer Architectures And Their Applications (Ijncaa), The Society Of Digital Information And Wireless Communications, vol. 2, no. 3, pp. 496-499, 2012.

[14] W. Y. Lin, M. W. Li, K. C. Lan, C. H. Hsu, "A Comparison of 802.11a and 802.11p for V-to-I Communication: A Measurement Study," in Proc. of Int. Conference on Heterogeneous Networking for Quality, Reliability, Security and Robustness, Berlin Heidelberg, Germany, 2010. Article (CrossRef Link)

[15] J. B. Kenney, "Dedicated Short-Range Communications (DSRC) Standards in the United States," in Proc. of the IEEE, vol. 99 July 2011. Article (CrossRef Link)

[16] E. M. v. Eenennaam, "A Survey of Propagation Models used in Vehicular Ad-hoc Network (VANET) Research,” a paper written for course Mobile Radio Communication, University of Twente, June 2008.

[17] S. Thelen, Connectivity Prediction in Mobile Ad Hoc Networks for Real-Time Control. Achen University, Books on Demand, 2015.

[18] S. Y. Wang, P. F. Wang, Y. W. Li, L.-C. Lau, "Design and Implementation of a More Realistic Radio Propagation Model for Wireless Vehicular Networks Over the NCTUns Network Simulator,” in Proc. of IEEE WCNC 2011 - PHY, pp. 1937-1942, 2011. Article (CrossRef Link)

[19] E. Giordano, R. Frank, G. Pau, M. Gerla, "CORNER: A Radio Propagation Model for VANETs in Urban Scenarios," in Proc. of the IEEE vol. 99, no. 7 July 2011. Article (CrossRef Link)

[20] S. A. H. Tabatabaei, M. Fleury, N. N. Qadri, M. Ghanbari, "Improving Propagation Modelling in Urban Environments for Vehicular Ad Hoc Networks," IEEE Transactions On Intelligent Transportation Systems, vol. 12, no. 3, pp. 705-716, September 2011. Article (CrossRef Link)

[21] R. L. Freeman, Fundamentals of Telecommunications, John Wiley \& Sons, Inc.

[22] Q. Sun, S. Y. Tan, K. C.Teh, "Analytical Formulae for Path Loss Prediction in Urban Street Grid Microcellular Environments,” IEEE Transactions On Vehicular Technology, vol. 54, no. 4, July 2005. Article (CrossRef Link)

[23] L. Denegri, "An Analytical Model of Microcellular Propagation in Urban Canyons," in Proc. of IEEE 65th Vehicular Technology Conference - VTC 2007-Spring, pp. 02-406 2007. Article (CrossRef Link)

[24] (2014). VisSim Comm. Available: http://www.vissim.com/products/vissim/comm.html

[25] (Apr 11, 2008, 25 April). WLAN - 802.11 a, b, g and $n$. Available: http://www.ni.com/tutorial/7131/en/

[26] R. Prasad, OFDM for Wireless Communications Systems. Boston - London, Artech House, Inc., 2004.

[27] R. Barr, Z. J. Haas, R. v. Renesse, "Jist: An efficient approach to simulation using virtual machines," Software Practice \& Experience, vol. 35, no. 6, pp. 539-576, 2005.

Article (CrossRef Link)

[28] R. Barr, Z. J. Haas, R. v. Renesse, "Chapter 19 - Scalable Wireless Ad Hoc Network Simulation,” in Proc. of Handbook on Theoretical and Algorithmic Aspects of Sensor, Ad hoc Wireless, and Peer-to-Peer Networks, ed Auerbach, 2005.

[29] C. Perkins, E. Royer, S. Das, "Ad hoc On-Demand Distance Vector (AODV) Routing, IETF RFC 3561,” 2003. 
[30] Radio theory and link planning for Wireless LAN (WLAN). Available: http://www.swisswireless.org/wlan_calc_en.html

[31] R. C. Poonia, V. Singh, "Performance Evaluation of Radio Propagation Model for Vehicular Ad hoc Networks Using VANETMOBISIM AND NS-2,” Int. Journal of Distributed and Parallel Systems (IJDPS) vol. 3, no. 4, July 2012. Article (CrossRef Link)

[32] A. Mukunthan, C. Cooper, F. Safaei, D. Franklin, M. Abolhasan, "Studying The Impact Of The Corner Propagation Model On Vanet Routing In Urban Environments," IEEE Vehicular Technology Conference, 2012. Article (CrossRef Link)

[33] E. Giordano, R. Frank, G. Pau, and M. Gerla, "CORNER: A Realistic Urban Propagation Model for VANET,” in Proc. of 7th IEEE/IFIP Conference on Wireless On demand Network Systems and Services (WONS 2010), Poster Session. Kranjska Gora, Slovenia: IEEE, pp. 57-60, February 2010. Article (CrossRef Link)

[34] A. I. Venkatesh and R. Murali, "Routing Protocols for Vehicular Adhoc Networks (VANETs): A Review,” Journal of Emerging Trends in Computing and Information Sciences, vol. 5, no. 1, pp. 56, 2014. Article (CrossRef Link)

[35] B. S. Kerner, "Failure of classical traffic flow theories: a critical review," Elektrotechnik und Informationstechnik, vol. 132, no. ,7 pp. 417-433, 2015. Article (CrossRef Link)

[36] R.V. Angeline, "Vehicular Ad-Hoc Networks: A Comprehensive Survey on Routing schemes," Int. Journal of Advanced Research in Education \& Technology (IJARET), vol. 2, no. 4, 2015. Article (CrossRef Link)

[37] J. Nzouonta, N. Rajgure, G. G. Wang, and C. Borcea, "VANET Routing on City Roads Using Real-Time Vehicular Traffic Information,” IEEE Transactions on Vehicular Technology, vol. 58 no. 7, pp. 3609-3626, 2009. Article (CrossRef Link)

[38] M. A. Qureshi, R. M. Noor, A. Shamim, S. Shamshirband, and K. K. Choo Raymond, "A lightweight radio propagation model for vehicular communication in road tunnels," PLoS ONE, vol. 11, no. 3, pp. 1-15, 2016. Article (CrossRef Link)

[39] J. A. Sanguesa, M. Fogue, P. Garrido, F. J. Martinez, J. C. Cano, and C. T. Calafate, “A Survey and Comparative Study of Broadcast Warning Message Dissemination Schemes for VANETs," Mobile Information Systems, vol. 2016, 2016. Article (CrossRef Link)

[40] W. Young, A. Sobhani, M.G. Lenne and M. Sarvi, "Simulation of safety: A review of state of the art in road safety simulation modelling,” Accident Analysis and Prevention, 66, pp. 89-103, 2014. Article (CrossRef Link)

[41] Friis, H. T., “A note on a simple transmission formula,” in Proc. of IRE, vol. 34, no. 5, pp. 254-256, 1946. Article (CrossRef Link)

[42] S. A. Soleymani, A. H. Abdullah, W. H. Hassan, M. H. Anisi, S.Goudarzi, M. A. R. Baee \&, S. Mandala, "Trust management in vehicular ad hoc network: a systematic review," EURASIP Journal on Wireless Communications and Networking, vol, no.1, pp. 146, 2015. Article (CrossRefLink)

[43] S. A. Soleymani, A. H. Abdullah, A. H., Anisi, M. H., A. Altameem, W. H. Hasan, S. Goudarzi, S. Manadala, A. B. Razak \& N. M. Noor. "BRAIN-F: Beacon Rate Adaption Based on Fuzzy Logic in Vehicular Ad Hoc Network,” International Journal of Fuzzy Systems, vol. 19, no. 2, pp. 301-315, 2017. Article (CrossRef Link)

[44] S. Goudarzi, A. H. Abdullah, S. Mandala, S. A. Soleymani, M. A. R.Baee, M. H. Anisi \& M. S. Aliyu, "A systematic review of security in vehicular Ad Hoc network," in Proc. of 2nd Symp. WSCN, pp. 1-10, 2013.

[45] S. Kalhor, M. Anisi \& A. T. Haghighat, “A new position-based routing protocol for reducing the number of exchanged route request messages in Mobile Ad-hoc Networks," in Proc. of Systems Second International Conference on and Networks Communications, 2007. ICSNC 2007. pp. 13-13, 2007. Article (CrossRef Link)

[46] S. S. Anjum, R. M. Noor, \& M. H. Anisi, "Survey on MANET based communication scenarios for search and rescue operations," in Proc. of 5th International Conference on IT Convergence and Security (ICITCS), pp. 1-5, 2015. Article (CrossRef Link) 
[47] M. Zareei, E. M, Mohamed, M. H. Anisi, C. V. Rosales, K. Tsukamoto \& M. K. Khan, “On-Demand Hybrid Routing for Cognitive Radio Ad-Hoc Network,” IEEE Access, vol 4, pp. 8294-8302, 2016. Article (CrossRef Link)

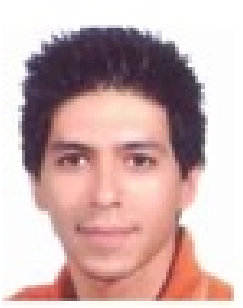

Ehsan Mostajeran received his BCS and MCS from University of Malaya, Kuala lumpur, Malaysia. He joined Malaysian National ICT research and development agency MIMOS Berhad in 2014. His research interests include radio propagations and routing protocols in Vehicular Ad hoc Network, Mobile Cloud Computing and Edge Computing.

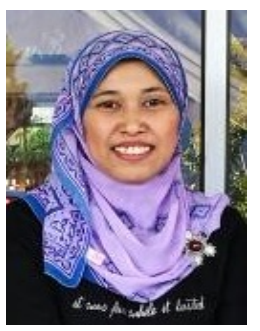

Rafidah Md Noor received her BIT from Universiti Utara Malaysia in 1998, MSc in Computer Science from Universiti Teknologi Malaysia in 2000, and $\mathrm{PhD}$ in Computing from Lancaster University, United Kingdom in 2010. She is currently an Associate Professor with the Department of Computer System and Technology, Faculty of Computer Science and Information Technology, University of Malaya and a Deputy Director for Centre of Mobile Cloud Computing Research (C4MCCR). Rafidah's research is related to field of transportation system in computer science domain, vehicular ad hoc networks, quality of service and Internet of Things.

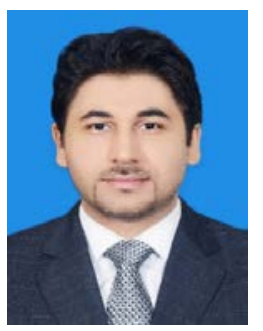

Mohammad Hossein Anisi is with the School of Computer Science and Electronics Engineering, University of Essex, United Kingdom. He received his Ph.D from Universiti Teknologi Malaysia (UTM) while being awarded as the best postgraduate student. He has worked as senior lecturer at the Faculty of Computer Science and Information Technology, University of Malaya. His research interests lie in the area of Internet of Things, wireless sensor networks and their applications, mobile Ad hoc networks, and intelligent transportation systems. He has also collaborated actively with researchers in several other disciplines of computer science. Dr. Anisi has published several papers in high quality journals and conferences. He is associate editor of the Ad Hoc \& Sensor Wireless Networks (SCIE) and the KSII Transactions on Internet and Information Systems (SCIE) journals. He is also active member of IEEE, ACM, International Association of Engineers (IAENG) and Institute of Research Engineers and Doctors (the IRED).

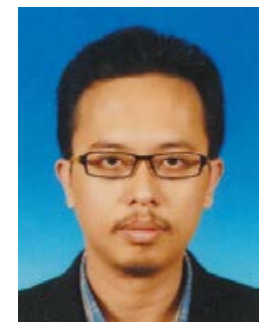

Ismail Ahmedy is a senior lecturer at the Department of Computer System and Technology, Faculty of Computer Science and Information Technology, University of Malaya, Kuala Lumpur. He received his BSc (Computer Science) from Universiti Teknologi Malaysia in 2006, MSc (Computer Science) from University of Queensland, Australia and $\mathrm{PhD}$ in Wireless Sensor Networks from Universiti Teknologi Malaysia, 2015. His research interest including WSN, Embedded System, underwater acoustic sensor networks and Internet of Things.

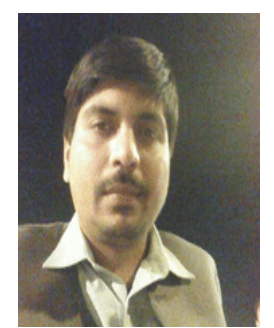

Fawad Ali Khan is a PhD candidate at the Department of Computer System and Technology, Faculty of Computer Science and Information Technology, University of Malaya. His research interests include Mobile Ad hoc Networks, Cloud Computing and Internet of Things. 